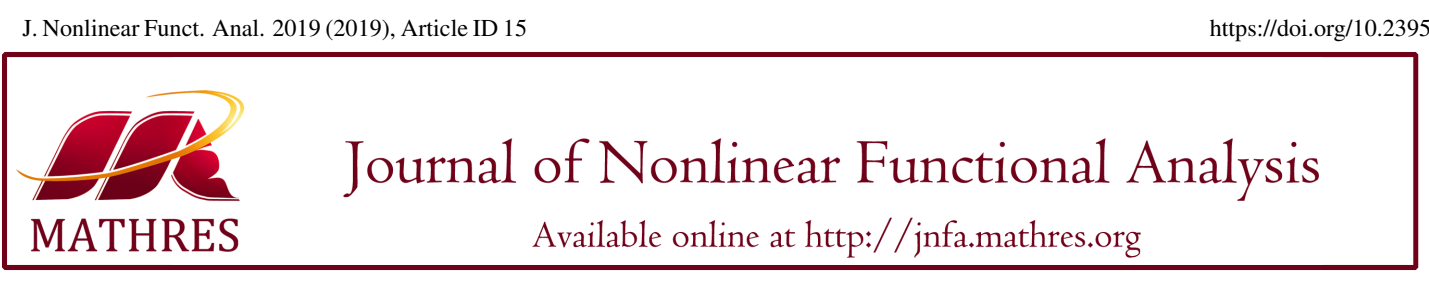

https://doi.org/10.23952/jnfa.2019.15

\title{
EXPANSIVE KRASNOSELSKII-TYPE FIXED POINT THEOREMS AND APPLICATIONS TO DIFFFERENTIAL INCLUSIONS
}

\author{
AHMED BOUDAOUI ${ }^{1, *}$, FATIMA BAHIDI $^{1}$, TOMÁS CARABALLO $^{2}$ \\ ${ }^{1}$ Department of Mathematics and Computer Science, University of Adrar, Adrar, Algeria \\ ${ }^{2}$ Departamento de Ecuaciones Diferenciales y Análisis Numérico, \\ Universidad de Sevilla, Tarfia s/n, Sevilla 41012, Spain
}

\begin{abstract}
In this paper, we prove some Krasnoselskii-type fixed point theorems in Banach spaces with the help of weak topologies. An example is provided to prove the existence of solutions for nonlinear integral inclusions in reflexive Banach spaces.
\end{abstract}

Keywords. Multivalued map; Nonlinear integral equation; Sequentially weakly continuous; Weakly compact; Fixed point.

2010 Mathematics Subject Classification. 47H10, 54H25.

\section{INTRODUCTION}

In 1958, Krasnosel'skii [1] proved the following fixed point theorem which is an important supplement to both the Banach contraction principle and the Schauder fixed point theorem.

Theorem 1.1. Let $\mathscr{M}$ be a nonempty closed convex subset of a Banach space $\mathscr{X}$. Suppose that $G$ and $D$ map $\mathscr{M}$ into $\mathscr{X}$ such that

(i) $G$ is continuous and $G(\mathscr{M})$ is contained in a compact subset of $\mathscr{X}$;

(ii) $D$ is a contraction with constant $\alpha<1$;

(iii) For any $u, v \in \mathscr{M}$ imply $D u+G v \in \mathscr{M}$.

Then there exists $u \in \mathscr{M}$ with $D u+G u=u$.

This is a significant result for solving nonlinear differential equations of the form:

$$
G u+D u=u .
$$

${ }^{*}$ Corresponding author.

E-mail addresses: ahmedboudaoui@gmail.com (A. Boudaoui), bahidifatima@gmail.com (F. Bahidi), caraball@us.es (T. Caraballo).

Received September 2, 2018; Accepted April 5, 2019.

(C) 2019 Journal of Nonlinear Functional Analysis 
Recently, some authors investigated Theorem 1.1 in the weak topology and some authors focused on the assumptions of Theorem 1.1; see, e.g., [2, 3, 4, 5, 6, 7, 8] and the references therein.

In [2], Barroso obtained a new version of Theorem 1.1 based on the weak topology of a Banach space. His result requires the weak continuity of $G$ while $D$ must be a linear operator with $\left\|D^{p}\right\|<1$ for some integer $p \geq 1$. The proof is based on the Schauder-Tychonoff fixed point theorem and the weak continuity of $(I-D)^{-1}$. In 2012, Garcia-Falset and Latrach [9] established a fixed point theorem for the sum $G+D$ of a weakly sequentially continuous mapping $G$ and a weakly sequentially continuous pseudocontractive, continuous and $w$-k-contraction $D$. Recently, Xiang and Yuan [10] formulated and proved some fixed point results of Krasnoselskii type for the sum $G+D$, where $G$ is weakly continuous and $D$ may not be continuous. Their results improved and unified many the previous ones. Moreover, the multivalued forms of Krasnoselskii's theorem deal with the notion of selection. Auxiliary results are needed to ensure that a multivalued operator admits a continuous selection. There is also a vast literature concerning these aspects in nonlinear analysis, see, for example, $[11,12,13]$ and the references therein.

In 2008, Boriceanu [12] established a Krasnoselskii's fixed point theorem for multivalued mappings which was used to solve the following inclusion:

$$
u \in G u+D u .
$$

Recently, many authors extended a number of existing generalizations or modifications of the multivalued forms of the Krasnosel'skii fixed point theorem for the weak topology; see, e.g., [9, 14, 15, 16] and the references therein.

In this paper, we introduce some single and multivalued versions of Krasnoselskii-type fixed point theorems which are important to analyze the situations that were not covered by previous literatures.

As an application of our main results, we discuss the existence of solutions of the nonlinear integral inclusion

$$
u(t) \in f(u(t))+\int_{0}^{t} B(s, x(s)) d s, t \in[0, T], T>0,
$$

in $C([0, T], \mathscr{X})$, where $\mathscr{X}$ is a reflexive Banach space, $f$ is function defined on $\mathscr{X}$ and $B$ is a multivalued mapping satisfying some conditions.

\section{PRELIMINARIES}

In this section, we introduce the notation and state some preliminary results which will be needed later in the paper.

Let $\mathscr{X}$ be a Hausdorff linear topological space. Define

$$
\begin{aligned}
\mathscr{P}(\mathscr{X}) & =\{\mathscr{Y} \subseteq \mathscr{X}: \mathscr{Y} \neq \emptyset\} \\
\mathscr{P}_{b}(\mathscr{X}) & =\{\mathscr{Y} \subseteq \mathscr{X}: \mathscr{Y} \text { bounded }\} \\
\mathscr{P}_{c v}(\mathscr{X}) & =\{\mathscr{Y} \subseteq \mathscr{X}: \mathscr{Y} \text { convex }\} \\
\mathscr{P}_{c p}(\mathscr{X}) & =\{\mathscr{Y} \subseteq \mathscr{X}: \mathscr{Y} \text { compact }\} \\
\mathscr{P}_{c l, c v}(\mathscr{X}) & =\{\mathscr{Y} \subseteq \mathscr{X}: \mathscr{Y} \text { closed and convex }\} \\
\mathscr{P}_{w k, c p}(\mathscr{X}) & =\{\mathscr{Y} \subseteq \mathscr{X}: \mathscr{Y} \text { weakly compact }\}, \\
\mathscr{P}_{w k, c p, c v}(\mathscr{X}) & =\{\mathscr{Y} \subseteq \mathscr{X}: \mathscr{Y} \text { weakly compact convex }\} .
\end{aligned}
$$

Now we suppose that $\mathscr{X}$ is a metrizable locally convex space and $\mathscr{M}$ is weakly closed in $\mathscr{X}$. 
Definition 2.1. Let $\mathscr{M}$ be a non-empty subset of a metrizable locally convex space $\mathscr{X}$ and let $D: \mathscr{M} \rightarrow$ $\mathscr{P}(\mathscr{X})$ be a multivalued mapping. $D$ is said to be weakly sequentially closed if for every sequence $\left(u_{n}\right)_{n} \subset \mathscr{M}$ with $u_{n} \rightarrow u$ in $\mathscr{M}$ and for every sequence $\left(v_{n}\right)_{n}$ with $v_{n} \in D\left(u_{n}\right), \forall n \in \mathbb{N}, v_{n} \rightarrow v$ in $\mathscr{X}$ implies $v \in D(u)$, where $\rightarrow$ denotes weak convergence.

Definition 2.2. Let $\mathscr{M}$ be a non-empty subset of a metrizable locally convex space $\mathscr{X}$ and let $D: \mathscr{M} \rightarrow$ $\mathscr{P}(\mathscr{X})$ be a multivalued mapping. $D$ is said to be weakly completely continuous if $D$ has a weakly sequentially closed graph and, if $F$ is a bounded subset of $\mathscr{M}$, then $D(F)$ is a relatively weakly compact subset of $\mathscr{X}$.

Theorem 2.3. [17] Let $\mathscr{M}$ be a closed and convex subset of $\mathscr{X}$ and let $D: \mathscr{M} \rightarrow \mathscr{P}_{c v}(\mathscr{M})$ be a multivalued mapping. Suppose that

(i) D is weakly completely continuous;

(ii) The set $D(\mathscr{M})$ is a bounded set of $\mathscr{X}$.

Then there exists $u \in \mathscr{M}$ such that $u \in D(u)$.

Definition 2.4. Let $(\mathscr{X}, d)$ be a metric space and let $\mathscr{M}$ be a subset of $\mathscr{X}$. The mapping $G: \mathscr{M} \rightarrow \mathscr{X}$ is said to be expansive if there exists a constant $h>1$ such that

$$
d(G u, G v) \geq h d(u, v), \quad \forall u, v \in \mathscr{M} .
$$

Lemma 2.5. [8] Let $(\mathscr{X},\|\cdot\|)$ be a linear normed space and $\mathscr{M} \subset \mathscr{X}$. Suppose that the mapping $G: \mathscr{M} \rightarrow \mathscr{X}$ is expansive with constant $h>1$. Then the inverse of $F$ exists, where $F:=I-G: \mathscr{M} \rightarrow$ $(I-G)(\mathscr{M})$ and

$$
\left\|F^{-1} u-F^{-1} v\right\| \leq \frac{1}{h-1}\|u-v\| \quad u, v \in F(\mathscr{M}) .
$$

Lemma 2.6. [8] Let $\mathscr{M}$ be a closed subset of a complete metric space $\mathscr{X}$. Assume that the mapping $G: \mathscr{M} \rightarrow \mathscr{X}$ is expansive and $G(\mathscr{M}) \supset \mathscr{M}$. Then there exists a unique point $u \in \mathscr{M}$ such that $G u=u$.

Lemma 2.7. [6] Let $G: \mathscr{X} \rightarrow \mathscr{X}$ be a map such that $G^{n}$ (n-power) is expansive for some $n \in \mathbb{N}$. Assume further that there exists a closed subset $\mathscr{M}$ of $\mathscr{X}$ such that $\mathscr{M} \subset G(\mathscr{M})$. Then there exists a unique fixed point of $G$ in $\mathscr{M}$.

Theorem 2.8. [18, Schauder-Tychonoff Theorem for the weak topology] Let $\mathscr{X}$ be a Banach space and let $\mathscr{M}$ be a weakly compact convex subset of $\mathscr{X}$. Then, any weakly continuous map $f: \mathscr{M} \rightarrow \mathscr{M}$ has at least one fixed point.

\section{THE EXPANSIVE KRASNOSEL'SKII-TYPE FIXED POINT THEOREM}

Let us now state and prove our first result for expansive mappings in the single-valued case.

Theorem 3.1. Let $\mathscr{X}$ be a Banach space and let $\mathscr{M}$ be a closed convex subset of $\mathscr{X}$. Let $G: \mathscr{M} \rightarrow \mathscr{X}$ be a weakly continuous map, and let $D \in \mathscr{L}(\mathscr{X})$ be a linear continuous operator. Assume that $D$ and $G$ satisfy the following hypotheses:

(i) $D$ is an expansive mapping,

(ii) For each $w \in G(\mathscr{M})$ we have $\mathscr{M} \subset D(\mathscr{M})+w$, where

$$
D(\mathscr{M})+w=\{v+w: v \in D(\mathscr{M})\},
$$


(iii) $G(M)$ is relatively weakly compact,

(iv) $D(u)+G(v) \in \mathscr{M}, \forall u, v \in \mathscr{M}$.

Then, equation $u=G(u)+D(u)$ possesses a solution in $\mathscr{M}$.

Proof. Let $v \in \mathscr{M}$. Let $F_{v}: \mathscr{M} \rightarrow \mathscr{X}$ be an operator defined by

$$
F_{v}(u)=D(u)+G(v), u \in \mathscr{M} .
$$

It is clear that $F_{v}$ is expansive. Then, by (ii) and Lemma 2.6, there exists a unique $u(v) \in \mathscr{M}$ such that

$$
u(v)=D(u(v))+G(v) .
$$

In view of assumptions (i), (iv) and Lemma 2.5, we obtain that

$$
u(v)=(I-D)^{-1} G(v) \in \mathscr{M} .
$$

Let us define

$$
\left\{\begin{aligned}
N: \mathscr{M} & \rightarrow \mathscr{M}, \\
v & \rightarrow N(v)=u(v),
\end{aligned}\right.
$$

which is weakly continuous since $(I-D)^{-1}$ and $G$ are weakly continuous. Let $\widetilde{\mathscr{M}}=\overline{c o}(N(\mathscr{M}))$ be the closed convex hull of $N(\mathscr{M})$. Using assumption (iii) and the Krein Šmulian theorem, we obtain that $\widetilde{\mathscr{M}}$ is a weakly compact and convex subset of $\mathscr{X}$.

Now, we only prove that $N(\widetilde{\mathscr{M}}) \subseteq \widetilde{\mathscr{M}}$. Indeed,

$$
\widetilde{\mathscr{M}}=\overline{c o}(N(\mathscr{M})) \subset \overline{c o}(\mathscr{M})=\mathscr{M} .
$$

Hence,

$$
N(\widetilde{\mathscr{M}}) \subset N(\mathscr{M}) \subset \overline{c o}(N(\mathscr{M}))=\widetilde{\mathscr{M}}
$$

Then, $N(\widetilde{\mathscr{M}}) \subseteq \widetilde{\mathscr{M}}$. Thanks to Theorem 2.8, there exists $u \in \widetilde{\mathscr{M}}$, which is a fixed point of $N$. Therefore, there exists $u \in \mathscr{M}$ such that $G u+D u=u$.

Due to Lemma 2.7, we can obtain the following result immediately.

Theorem 3.2. Let $\mathscr{X}$ be a Banach space and let $\mathscr{M}$ be a closed convex subset of $\mathscr{X}$. Let $G: \mathscr{M} \rightarrow \mathscr{X}$ be a weakly continuous map and let $D \in \mathscr{L}(\mathscr{X})$ be a linear continuous operator. Assume that $D$ and $G$ satisfy the conditions of Theorem 3.1, but replacing condition (i) by the fact that $D^{n}$ is expansive. Then, equation $u=G(u)+D(u)$ possesses a solution in $\mathscr{M}$.

\section{THE KRASNOSEL'SKII THEOREM FOR MULTIVALUED OPERATORS}

Now, we establish our first result for multivalued operators.

Theorem 4.1. Let $(\mathscr{X},\|\|$.$) be a Banach space and let \mathscr{M}$ be a closed and convex subset of $\mathscr{X}$. Assume that $G: \mathscr{M} \rightarrow \mathscr{P}_{w k, c p, c v}(\mathscr{X})$ is weakly sequentially continuous and $D \in \mathscr{L}(\mathscr{X})$ satisfies

(i) $D$ is expansive,

(ii) For each $w \in G(\mathscr{M})$ we have $\mathscr{M} \subset D(\mathscr{M})+w$, where

$$
D(\mathscr{M})+w=\{v+w: v \in D(\mathscr{M})\} .
$$

Then there exists $u \in \mathscr{M}$ such that $u \in G(u)+D(u)$. 
Proof. For each $v \in \mathscr{M}$, let $F_{v}: \mathscr{M} \rightarrow \mathscr{P}(\mathscr{X})$ be the multivalued operator defined by

$$
F_{v}(u)=D(u)+G(v), \quad u \in \mathscr{M} .
$$

From Lemma 2.6, there exists a unique $u(v) \in \mathscr{M}$ such that

$$
u(v) \in D(u(v))+G(v) .
$$

By (i), we obtain that

$$
u(v) \in(I-D)^{-1} G(v) .
$$

Since $G(v) \in \mathscr{P}_{w k, c p, c v}(\mathscr{X})$, the multivalued operator $N: \mathscr{M} \rightarrow \mathscr{P}_{w k, c p, c v}(\mathscr{X})$ defined by $N(v)=(I-$ $D)^{-1} G(v)$ is well defined.

Now, we show that $N$ is weakly completely continuous. Let $\left(u_{n}\right)_{n} \subset \mathscr{M}$ and $v_{n} \in N\left(u_{n}\right)$ such that $u_{n} \rightarrow u$ in $\mathscr{M}$ and $v_{n} \rightarrow v$ in $\mathscr{X}$. It is easy to prove that

$$
(I-D)^{-1} \in \mathscr{L}(\mathscr{X}) .
$$

Thus, $(I-D)^{-1}$ is weakly continuous. Then $v_{n} \rightarrow \widetilde{v} \in(I-D)^{-1} G(u)$ by uniqueness $v=\widetilde{v} \in N(u)$. This prove that $N$ has a weakly sequentially closed graph.

Let $\mathscr{K}$ be a bounded set in $\mathscr{M}$. We next prove that $N(\mathscr{K})$ is relatively weakly compact. To see that, let $u_{n} \in \mathscr{K}$ and $v_{n} \in N\left(u_{n}\right)$. There exists $\left(w_{n}\right)_{n} \in G\left(v_{n}\right)$ such that $v_{n}=(I-D)^{-1} w_{n}$ or, equivalently, $w_{n}=$ $(I-D) v_{n}$. Since $G(v)$ is weakly compact, there exists $w \in G(v)$ such that $w_{n} \rightarrow w$. This implies that $v_{n} \rightarrow$ $(I-D)^{-1} w$. This prove that $N(\mathscr{K})$ is sequentially relatively compact. By Eberlein Šmulian theorem [19, Theorem 3.14], $N(\mathscr{K})$ is relatively weakly compact. Thus $N$ is weakly completely continuous.

On the other hand, $N(\mathscr{M}) \subset \mathscr{P}_{w k, c p, c v}(\mathscr{X})$. Consequently, $N(\mathscr{M})$ is a bounded set of $\mathscr{X}$. By Theorem 2.3, we conclude that there exists $u \in \mathscr{M}$ such that $u \in N(u)$.

Finally, we prove our last result in this paper.

Theorem 4.2. Let $(\mathscr{X},\|\|$.$) be a Banach space and let \mathscr{M}$ be closed and convex subset of $\mathscr{X}$. Assume that $G: \mathscr{M} \rightarrow \mathscr{P}_{\text {wk,cp,cv}}(\mathscr{X})$ is sequentially weakly continuous and $D \in \mathscr{L}(\mathscr{X})$ satisfies

(i) $D$ is expansive and onto,

(ii) $(I-D)(\mathscr{M}) \subset G(\mathscr{M})$.

Then there exists $u \in \mathscr{M}$ such that $u \in G(u)+D(u)$.

Proof. Let $v \in \mathscr{M}$. From (ii), there exists $u \in \mathscr{M}$ such that $(I-D)(u) \in G(v)$. From (i), we can prove that $(I-D)^{-1}$ exists and $u \in(I-D)^{-1} G(v)$. Using the same technique as that in Theorem 4.1, we find $u \in \mathscr{M}$ such that $u \in G(u)+D(u)$. This completes the proof.

\section{APPLICATIONS TO NONLINEAR INTEGRAL EQUATIONS}

We provide now an example for which we can prove the existence of solutions of nonlinear integral inclusion (5.1) under conditions $\left(H_{1}\right)-\left(H_{5}\right)$ below. However, it is worth mentioning that the same result cannot be guaranteed by previous results in the literatures under assumptions $\left(H_{1}\right)-\left(H_{5}\right)$.

Let $\mathscr{X}$ be a reflexive Banach space. The objective of this section is to prove the existence of solutions for the following nonlinear integral inclusion

$$
u(t) \in f(u(t))+\int_{0}^{t} B(s, u(s)) d s, \quad t \in[0, T], T>0,
$$


in $C([0, T], \mathscr{X}), f: \mathscr{X} \rightarrow \mathscr{X}$ and $B:[0, T] \times \mathscr{X} \rightarrow P_{c v}(\mathscr{X})$ and $u \in \mathscr{X}$. We pose

$$
\|B(t, u)\|=\sup \{|x|, x \in B(t, u)\} .
$$

The integral in (5.1) is the Pettis integral and the solutions of (5.1) are in Banach space $\mathscr{E}=C([0, T], \mathscr{X})$. We consider (5.1) inclusion under the following assumptions.

$\left(H_{1}\right) f \in \mathscr{L}(\mathscr{X})$ is expansive and onto;

$\left(H_{2}\right)$ For each continuous function $u:[0, T] \rightarrow \mathscr{X}$ there exists a function $y:[0, T] \rightarrow \mathscr{X}$ weakly continuous with $y(t) \in B(t, u(t))$ for all $t \in[0, T]$, and $y$ is Pettis integrable;

$\left(H_{3}\right)$ There exists $r>0$, and $h_{r} \in L_{1}([0, T])$ with $\|B(t, u)\| \leq h_{r}(t)$ for a.e. $t \in[0, T]$ and all $x \in \mathscr{X}$ with $|x| \leq r$. We let

$$
M_{r}=\int_{0}^{t} h_{r}(s) d s \leq r
$$

$\left(H_{4}\right)$ For all $t \in[0, T], B(t, \cdot)$ is weakly completely continuous;

$\left(H_{5}\right)$ For each $v \in(I-f)(\mathscr{M})$ there exist $u \in \mathscr{M}$ and $y(s) \in B(s, u(s))$ pettis integrable such that

$$
v(t)=\int_{0}^{t} y(s) d s .
$$

Theorem 5.1. Assume that hypotheses $\left(H_{1}\right)-\left(H_{5}\right)$ hold. Then nonlinear integral inclusion (5.1) has a solution in $C([0, T], \mathscr{X})$.

Proof. Inclusion (5.1) can be written in the form

$$
u(t) \in G u(t)+D u(t)
$$

where

$$
\begin{aligned}
& G: \mathscr{E} \rightarrow \mathscr{P}(\mathscr{E}), G u(t)=\left\{x(t)=\int_{0}^{t} y(s) d s, y \text { is Pettis integrable, } y(t) \in B(t, u(t))\right\} \\
& D: \mathscr{E} \rightarrow \mathscr{E}, D u(t)=f(u(t)) .
\end{aligned}
$$

Let

$$
\mathscr{M}=\{u \in \mathscr{X},\|u\| \leq r\} .
$$

It is clear that $\mathscr{M}$ is convex closed subset of $\mathscr{X}$. We next show that mappings $G$ and $D$ satisfy all the conditions of Theorem 4.2.

Step 1. $D \in L(\mathscr{X})$ is expansive and onto.

Step 2. We prove that $G u \in \mathscr{P}_{w k, c p, c v}(C([0, T], \mathscr{X}))$ for all $u \in \mathscr{E}$. We show that $G(u)$ is convex, bounded and closed subset of $\mathscr{X}$. Let $u, u^{\prime} \in \mathscr{E}$ and $v, v^{\prime} \in G(u)$ such that

$$
\begin{gathered}
v(t)=\int_{0}^{t} y(s) d s, \\
v^{\prime}(t)=\int_{0}^{t} y^{\prime}(s) d s,
\end{gathered}
$$

where $y, y^{\prime}$ are Pettis integrable with $y(s), y^{\prime}(s) \in B(s, u(s))$ for all $s \in[0, T]$. Letting $\lambda \in[0,1]$, we have

$$
\lambda v(t)+(1-\lambda) v^{\prime}(t)=\int_{0}^{t}\left(\lambda y(s)+(1-\lambda) y^{\prime}(s)\right) d s .
$$


Since $B(t, u(t))$ is a convex subset of $\mathscr{X}$, one has $\left(\lambda y(s)+(1-\lambda) y^{\prime}(s)\right) \in B(t, u(t))$, which proves that $G(u)$ is convex. Let $u \in \mathscr{M}$. By the Hahn-Banach Theorem, there exists $\varphi \in \mathscr{X}^{*}$ such that $\|\varphi\|=1$ and $\varphi(G u(t))=\|G(u(t))\|$

$$
\begin{aligned}
\|G(u(t))\| & =\int_{0}^{t} y(s) d s \\
& \leq \int_{0}^{t}\|B(s, u(s))\| d s \\
& \leq \int_{0}^{t} h_{r}(s) d s \leq r .
\end{aligned}
$$

This proves that $G(u) \in \mathscr{M}$. So, it is a closed and bounded set. Since $\mathscr{X}$ is reflexive, one concludes that $G(u)$ is weakly compact.

Step 3. $G$ is weakly sequentially continuous. Let $u_{n} \in \mathscr{X}$ such that $u_{n} \rightarrow u$. We prove that $G u_{n} \rightarrow G u$.

Let $v_{n} \in G\left(u_{n}\right)$ such that $v_{n} \rightarrow v, v \in \mathscr{X}$. There exists $y_{n}(t) \in B\left(t, u_{n}(t)\right)$ such that

$$
v_{n}(t)=\int_{0}^{t} y_{n}(s) d s
$$

By assumption $\left(H_{2}\right)$, there exist $y(s) \in B(t, u(t))$ such that

$$
v_{n}(t)=\int_{0}^{t} y_{n}(s) d s \rightarrow \int_{0}^{t} y(s) .
$$

By the uniqueness of the weak limit and assumption $\left(H_{4}\right)$, we have

$$
v(t)=\int_{0}^{t} y(s) \in G(u) .
$$

Then $v_{n}(t) \rightarrow v(t)$. By the Dobrokov's Theorem [20, Theorem 1.4.1], we prove that $G$ is sequentially weakly continuous. Then $G$ and $D$ satisfy the conditions of Theorem 4.2. So, equation (5.1) has a solution in $C([0, T], \mathscr{X})$. This completes the proof.

\section{Acknowledgement}

The authors are grateful to the referees for the careful reading and the helpful remarks.

\section{REFERENCES}

[1] M.A. Krasnoselskii, Some problems of nonlinear analysis, Amer. Math. Soc. Transl. 10 (1958), 345-409.

[2] C.S. Barroso, Krasnoselskii's fixed point theorem for weakly continuous maps, Nonlinear Anal. 55 (2003), 25-31.

[3] C.S. Barroso, C.S. Barroso, E.V. Teixeira, A topological and geometric approach to fixed points results for sum of operators and applications, Nonlinear Anal. 60 (2005), 625-650.

[4] T. A. Burton, Krasnoselskii's inversion principle and fixed points, Nonlinear Anal. 30 (1997), 3975-3986.

[5] T. A. Burton, C. Kirk, A fixed point theorem of Krasnoselskii-Schaefer type, Math. Nachr. 189 (1998), 23-31.

[6] L. Górniewicz, A. Ouahab, Some fixed point theorems of a Krasnoselskii type and application to differential inclusions, Fixed Point Theory. 17 (2016), 85-92.

[7] Y. Liu, Z. Li, Krasnosel'skii-type fixed point theorems, Proc. Amer. Math. Soc. 136 (2008), 1213-1220.

[8] T. Xiang, R. Yuan, A class of expansive-type Krasnoselskii fixed point theorems, Nonlinear Anal. 71 (2009), $3229-3239$.

[9] J. Garcia-Falset, K. Latrach, Krasnoselskii-type fixed point theorems for weakly sequentially continuous mappings, Bull. London Math. Soc. 44 (2012), 25-38.

[10] T. Xiang, R. Yuan, Krasnoselskii-type fixed point theorems under weak topology settings and applications, Electron. J. Differential Equations 35 (2010), 1-15. 
[11] J. Andres, L. Górniewicz, Topological Fixed Point Principles for Boundary Value Problems, Kluwer, Dordrecht, (2003).

[12] M. Boriceanu, Krasnoselskii-type theorems for multivalued operators, Fixed Point Theory 9 (2008), 35-45.

[13] L. Górniewicz, Topological Fixed Point Theory of Multi-valued Mappings, 2nd ed., Springer, (2006).

[14] R. P. Agarwal, D. O’Regan, M. A. Taoudi, Fixed point theorems for condensing multivalued mappings under weak topology features, Fixed Point Theory 12 (2011), 247-254.

[15] A. A. Alsheikh, A. Ben Amar, D. O'Regan, Fixed point theorems for the sum of two multivalued mappings and an application to an integral inclusion, Bull. Malaysian Math. Sci. Soc. 40 (2017), 1307-1320.

[16] A. Ben Amar, M. Boumaiza, D. O'regan, Hybrid fixed point theorems for multivalued mappings in Banach algebras under a weak topology setting, J. Fixed Point Theory Appl. 18 (2016), 327-350.

[17] A. Ben Amar, T. Xiang, Critical types of Krasnoselskii fixed point theorems in weak topologies, Quaestiones Math. 38 (2015), 805-816.

[18] Z. Sahnoun, Théorie du point fixe pour les sommes et produits d'opérateurs dans des espaces localement convexes, et applications, E.N.S-Kouba, Juin 2011, Encardé par, DJEBALI Smail.

[19] K. Qarri, Eberlein-Šmulian theorem and some of its applications, University of Agder, Faculty of Engineering and Science, Department of Mathematics, Master Thesis in Mathematics Education, (2014).

[20] A. Jeribi, B. Krichen, Nonlinear functional analysis in Banach spaces and Banach algebras: fixed point theory under weak topology for nonlinear operators and block operator matrices with applications, CRC Press, New York. (2015). 